\title{
A capacitação tecnológica na empresa: a função da Tecnologia Industrial Básica (TIB)
}

\author{
Technological capability development: the role of \\ Infrastructural Technology
}

\author{
Renato Gallina ${ }^{1}$ \\ Afonso Fleury ${ }^{2}$
}

\begin{abstract}
Resumo: O domínio da Tecnologia Industrial Básica - TIB - (metrologia, normalização e avaliação da conformidade) não é somente um requisito para a competitividade de uma empresa por possibilitar que esta produza em conformidade com as normas internacionais, mas é também uma condição necessária para a efetiva acumulação de capacidades tecnológicas e a busca da inovação tecnológica. Na literatura especializada, raros são os estudos que analisam a função da TIB no processo de aprendizagem tecnológica. Este trabalho se propõe a preencher essa lacuna ao propor um modelo para avaliar e projetar as relações dinâmicas entre TIB e capacidade tecnológica. Esse modelo permite cotejar os mecanismos utilizados em cada um dos estágios de capacitação em TIB com os processos organizacionais adotados por empresas situadas nos diferentes níveis de capacitação tecnológica identificados na literatura. A coleta de dados foi feita por um survey, que envolveu uma amostra de 103 empresas do setor metal-mecânico. Concluiu-se que há uma forte relação entre o nível de capacitação em TIB e o nível de capacitação tecnológica das empresas. Em outras palavras, o desenvolvimento da capacitação em TIB é uma pré-condição para o desenvolvimento da capacitação tecnológica das empresas.
\end{abstract}

Palavras-chave: Tecnologia Industrial Básica (TIB). Capacidade tecnológica. Setor metal-mecânico. Metrologia. Normalização. Avaliação de conformidade.

\begin{abstract}
The mastering of Infrastructural Technologies (IfT) - metrology, standardization, and conformity assessment - is not only a precondition for a firm's competitiveness, since it enables production in accordance with international norms; but it is also a requisite for the effective accumulation of technological capabilities aiming at technological innovations. In the specialized literature, there is a lack of studies that address the role of Infrastructural Technologies in the technology learning process. This article aims to fill that gap by proposing a model to assess and design the dynamic interrelationships between IfT and technological capability development. This model compares the mechanisms required in each one of the stages of IfT maturity to the organizational processes adopted by firms with different levels of technological capability reported in the literature. The data were collected through a survey of a sample of 103 metal-mechanics firms. It can be concluded that there is a strong relationship between the IfT's level of maturity and the firms' level of technological capability. In other words, the development of capability in IfT seems to be a precondition for the firms' technological development and innovation strategy.
\end{abstract}

Keywords: Infrastructural Technologies. Technological capability. Metal-mechanics sector. Metrology. Standardization. Conformity assessment.

\section{Introdução}

Um relatório produzido pelo Banco Mundial intitulado "Trade Facilitation and Economic Growth: The Development Division" destaca que iniciativas para acelerar o comércio internacional por meio da remoção de barreiras não tarifárias, entre outras, são reconhecidas, atualmente, como fatores que afetam o desempenho da indústria. O relatório ressalta que isto é particularmente importante para as empresas

de menor porte em países em desenvolvimento. A habilidade das empresas em entregar produtos e serviços no prazo, com o menor custo possível e em conformidade com as normas, é fator determinante para ingressar na economia global.

Enquanto um significante declínio em barreiras explícitas ao comércio tem sido observado nas últimas décadas, normas e regulamentos técnicos são utilizados

\footnotetext{
Centro Universitário da FEI, Av. Humberto de Alencar Castelo Branco, 3972, CEP 09850-901, São Bernardo do Campo, SP, e-mail: gallina@fei.edu.br

${ }^{2}$ Departamento de Engenharia de Produção, Escola Politécnica, Universidade de São Paulo - USP, Av. Professor Almeida Prado,

128, travessa 2, CEP 05508-900, São Paulo, SP, e-mail: acfleury @usp.br
} 
com maior frequência alterando a lógica das transações comerciais. Isto é particularmente verdadeiro para as empresas em países em desenvolvimento. Estudos conduzidos pela United Nations Conference on Trade and Development - UNCTAD demonstram que alguns países em desenvolvimento vêm sofrendo consideráveis perdas em suas exportações pela dificuldade de atender a normas e regulamentos ambientais restritivos e duplicados, impostos por países desenvolvidos.

Para as empresas dos países em desenvolvimento cumprirem um número crescente de normas e regulamentos técnicos relacionados ao conteúdo dos produtos, são necessárias mudanças em métodos e processos de produção. Além disso, várias legislações sobre rastreabilidade surgiram nos últimos anos e acabaram se constituindo em barreiras ao comércio, pois exigem a implementação de sofisticados sistemas, demandando grandes investimentos. Essas empresas, por sua menor capacidade tecnológica, têm maior dificuldade de se adaptar e seguir as regras estipuladas (NADVI; WALTRING, 2002). Segundo Fleury (2003), esse é um processo marcado por inúmeras dificuldades, uma vez que, por trás de todo esse esforço, trata-se de modificar os sistemas produtivos. Por exemplo, a qualidade dos produtos passou a ser exigida de forma mais objetiva e explícita: objetiva por ter necessariamente que estar lastreada em normas técnicas ou em regulamentos técnicos, de preferência internacionais; explícita na medida em que a adequação a tais normas tem que estar comprovada por medições, inspeções, ensaios, testes e certificações.

Para tornar essas empresas competitivas, a aprendizagem tecnológica torna-se de fundamental importância (BELL; PAVITT, 1993). Os processos de aprendizado são associados à ampliação do estoque de conhecimentos, ao aprimoramento contínuo, a processos de inovação, ao desenvolvimento de competências, à obtenção de diferenciais em relação aos concorrentes, implicando aumento da capacidade competitiva das empresas.

Segundo Fransman (1984), os estudos sobre países em desenvolvimento identificaram uma mudança, a partir da década de 1980, das noções de transferência de tecnologia e de escolhas passivas para o aprendizado tecnológico. Esta mudança tem característica endógena, cumulativa e tácita: a empresa acumula sequencialmente conhecimentos, experiências e aptidões que lhe permitem evoluir da mera operação de determinada tecnologia, para a busca, absorção, melhoramento e modificações substanciais até atingir um nível de domínio da tecnologia que lhe permita realizar inovações e operar na fronteira internacional do conhecimento tecnológico, realizando assim o catch-up (FRANSMAN, 1984; FLEURY, 1991; LALL, 1992; FIGUEIREDO, 2004).

Nesse contexto, a normalização e a metrologia tornam-se temas estratégicos. O eventual desconhecimento da importância desses temas e a não aplicação de seus princípios significarão enormes perdas de competitividade.

Assim, o domínio das funções tecnológicas da Tecnologia Industrial Básica - TIB - (metrologia, normalização e avaliação da conformidade) torna-se não somente um requisito para a competitividade de uma empresa, mas também a base para a efetiva acumulação de capacidades tecnológicas necessárias para o melhoramento contínuo e a inovação em produtos e serviços. A tese defendida neste trabalho é que o desenvolvimento das empresas em termos de capacitação tecnológica é associado e suportado pelo avanço em metrologia, normalização e avaliação da conformidade.

Na literatura especializada, raros são os estudos que analisam a função da TIB no processo de aprendizagem tecnológica de uma empresa. Este trabalho tem a intenção de preencher essa lacuna ao propor um modelo para avaliar e projetar as relações dinâmicas entre TIB e capacidade tecnológica, respondendo às seguintes questões:

- As competências conquistadas no âmbito da Tecnologia Industrial Básica (TIB) contribuem efetivamente para $o$ incremento da capacidade tecnológica das empresas? Quais as principais relações, se é que existem, que podem ser estabelecidas entre TIB e capacidade tecnológica?

- Os mecanismos de aprendizagem em TIB variam durante o seu processo de formação e acumulação de competências?

$\mathrm{O}$ foco deste trabalho está no relacionamento entre as funções tecnológicas da TIB e a acumulação de capacidades tecnológicas na empresa, como mostrado na Figura 1.

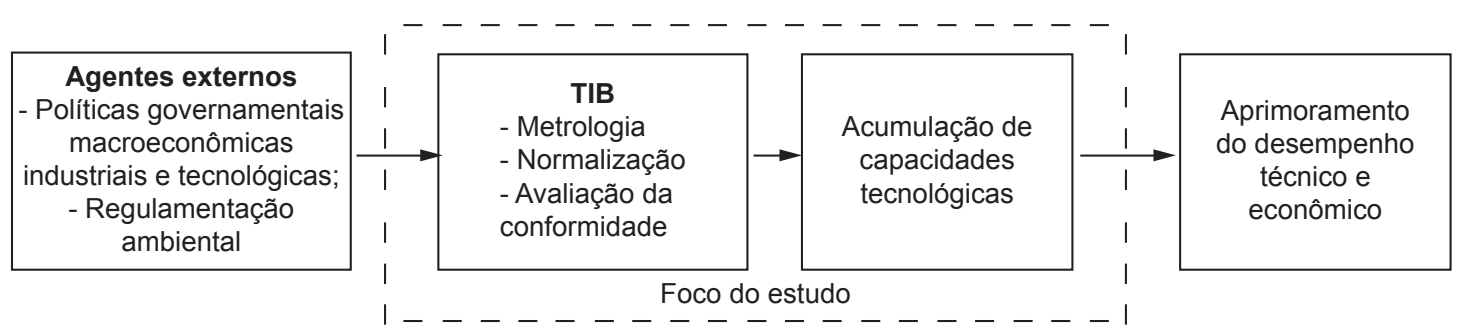

Figura 1. Sequência de ações envolvendo TIB e Capacidade Tecnológica. Fonte: adaptado de Tacla e Figueiredo (2003). 
Acredita-se que o presente trabalho será útil no sentido de contribuir para a análise da TIB no ambiente empresarial, ao revelar as relações mais significativas entre TIB e capacidade tecnológica, bem como o papel da TIB na formação da capacidade tecnológica das empresas.

O estudo foi realizado por meio de um survey, cujo instrumento de pesquisa foi um questionário aplicado em uma amostra de 103 empresas de diferentes níveis de capacidade tecnológica do setor metal-mecânico. Para se chegar aos resultados desta pesquisa, foram utilizadas as técnicas estatísticas da correlação canônica e de análise de correspondência ANACOR para se concluir sobre as relações significativas entre TIB e capacidade tecnológica, bem como sobre a utilização dos diversos mecanismos de aprendizagem em TIB, respectivamente.

Este artigo está estruturado da seguinte forma: no item 1 ( introdução), está descrito o tema do trabalho de pesquisa, sua importância, a questão da pesquisa, a tese, a contribuição teórica e a metodologia utilizada. No item 2 (referenciais teóricos), apresenta-se uma síntese da revisão bibliográfica acerca dos temas: aprendizagem/capacidade tecnológica e Tecnologia Industrial Básica. No item 3 (a construção do modelo de análise), apresentam-se a síntese do desenvolvimento de um referencial analítico sobre capacitação em TIB, a qual serviu de base para a elaboração do modelo conceitual da pesquisa, bem como os principais mecanismos de aprendizagem para a formação e acumulação de competências em TIB. No item 4 (metodologia de pesquisa), são apresentadas as variáveis, os indicadores, a construção das proposições e o método de coleta de informações. No item 5 (resultados), são apresentados e discutidos os resultados da pesquisa obtidos a partir da aplicação das técnicas estatísticas ao conjunto de dados obtidos no survey. No item 6 (conclusões), são enfatizadas as principais conclusões deste trabalho de pesquisa.

\section{Referenciais teóricos}

Neste trabalho são consideradas as seguintes definições:

- Capacidade tecnológica (ou competência tecnológica): o conjunto dos recursos necessários tanto para usar certa tecnologia com determinado nível de eficiência como para gerar e gerir mudanças tecnológicas; tais recursos se acumulam e se incorporam aos indivíduos em termos de aptidões, conhecimento e experiência, bem como aos sistemas organizacionais (BELL; PAVITT, 1993, 1995);

- Processos de aprendizagem tecnológica: processos pelos quais as pessoas e, por meio delas, as organizações adquirem aptidões e conhecimentos técnicos (BELL, 1984), ou seja, os processos pelos quais a aprendizagem individual se converte em aprendizagem organizacional. Os processos de aprendizagem permitem à empresa acumular competência tecnológica ao longo do tempo. Considera-se que a empresa acumulou um determinado nível de competência quando ela se torna apta a alguma atividade tecnológica que não podia desempenhar anteriormente (FIGUEIREDO, 2003);

- Tecnologia Industrial Básica (TIB): o conjunto das funções básicas de metrologia, normalização e avaliação da conformidade - ensaios, inspeções e certificações (SOUZA, 2000).

Como referência principal para a análise dos estágios de capacitação tecnológica foi adotada a matriz de Lall (1992), apresentada no Quadro 1, que trata da evolução das capacidades tecnológicas para as empresas de países em desenvolvimento. $\mathrm{O}$ autor identifica três graus de complexidade, segundo a formalidade e o propósito dos esforços tecnológicos: básico, intermediário e avançado.

As capacidades tecnológicas básicas são acumuladas por meio da experiência e das rotinas básicas da atividade de produção, ou seja, é do tipo learning by-doing ou com base na experiência. As capacidades intermediárias são construídas a partir de atividades ou esforços conduzidos em base mais deliberada. E as capacidades avançadas, por sua vez, são desenvolvidas por meio de atividades de $\mathrm{P} \& \mathrm{D}$, que são a forma mais explícita e deliberada de esforço tecnológico.

\section{A construção do modelo de análise}

A necessidade de se criar um referencial analítico que permitisse avaliar o nível de capacidade em TIB de uma empresa surgiu a partir da constatação da não existência de referências na literatura especializada. Para a elaboração desse referencial analítico, foram consideradas as seguintes premissas:

- Perspectiva evolucionista: a acumulação das capacidades em TIB se processa das categorias mais simples para as mais complexas. Foram considerados seis níveis de capacidade: primário, básico (perfil 5 - PF5), extrabásico (perfil 4 - PF4), intermediário (perfil 3 - PF3), intermediário superior (perfil 2 - PF2) e avançado (perfil 1 - PF1). Esses níveis foram identificados a partir de uma análise evolucionista na definição e implantação de normas, iniciada com as de primeira geração (norma genérica - ISO 9000), seguiram-se normas de segunda e terceira gerações (de setor específico e de empresas), passando pelas normas ambientais, sociais e 


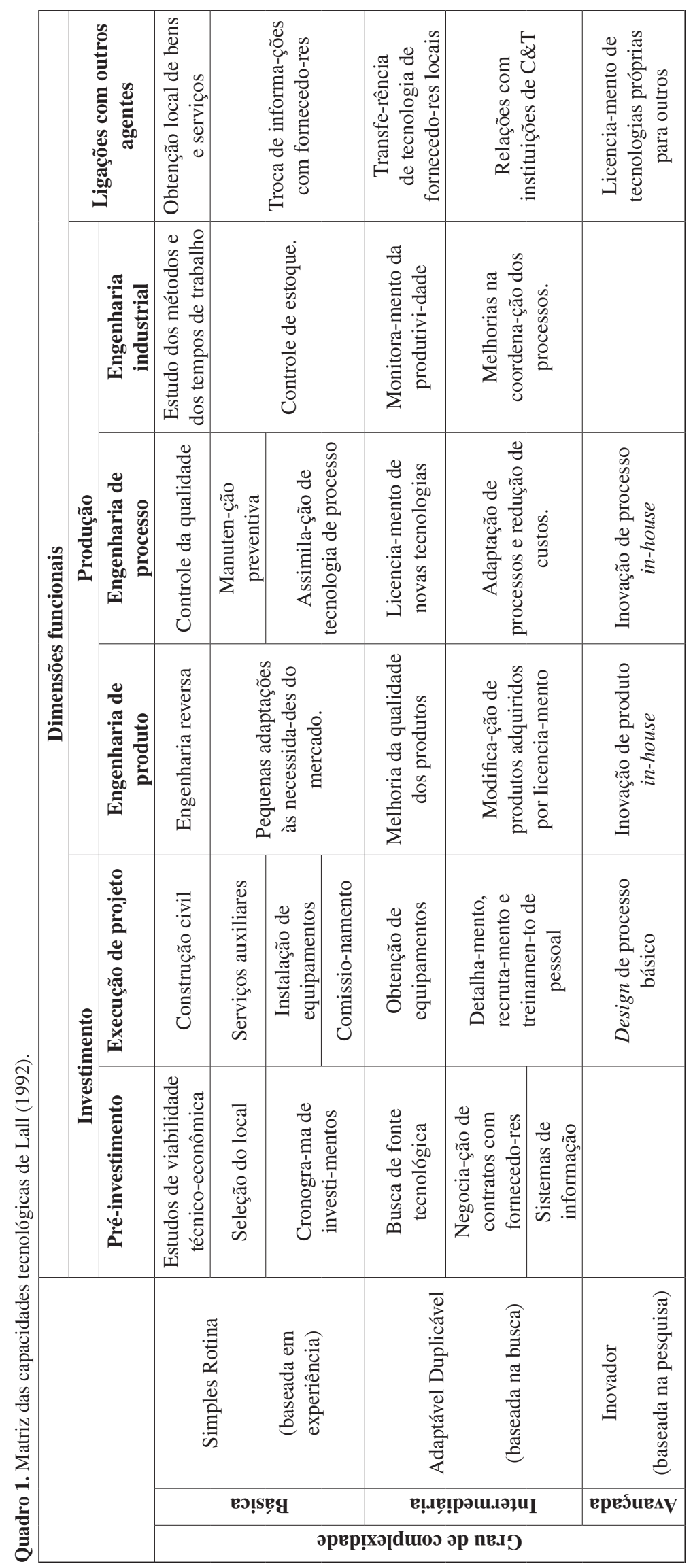


admitindo, como nível mais alto, a capacidade que empresas possam vir a ter na formulação e uso de normas globais;

- A distinção entre os diferentes tipos de capacitação em TIB - metrológico, normativo e de avaliação da conformidade.

O Quadro 2 caracteriza os níveis de capacitação em TIB nas três funções tecnológicas básicas.

Em seguida, a partir de uma série de informações sobre mecanismos de aprendizagem encontradas de forma dispersa na literatura especializada, foram identificados os principais mecanismos organizacionais adotados nas empresas situadas nos diferentes níveis da escala antes mencionada, como mostrado na Figura 2. Os mecanismos de aprendizagem estão colocados entre os diversos perfis de empresas para salientar a influência deles na evolução das competências em TIB. Conforme relatado anterior, as empresas de nível primário praticamente não têm capacitação em TIB.

\section{Metodologia de pesquisa}

\subsection{Definição de variáveis, indicadores e construção das proposições}

Para analisar a relação entre a TIB e a formação e acumulação das capacidades tecnológicas das empresas, foi desenvolvida a análise das relações entre a variável TIB (classificada neste trabalho como independente) e a variável capacidade tecnológica (classificada neste trabalho como dependente), por seus indicadores, conforme mostra o Quadro 3.

As proposições $\mathrm{P} 1, \mathrm{P} 2$ e $\mathrm{P} 3$ assinaladas no Quadro 3 correlacionam os indicadores da variável independente aos da variável dependente.

P1: uma empresa que está no nível básico de capacitação em TIB (PF5 e PF4 por serem certificadas pela ISO 9001 (INTERNATIONAL..., 2008) e/ ou TS16949 (INTERNATIONAL..., 2009)) adota processos organizacionais relacionados à capacitação tecnológica (engenharia reversa, poucos esforços de adaptação do produto ao mercado, etc.) que revelam baixa capacitação tecnológica.

P2: uma empresa que está num nível intermediário de capacitação tecnológica em TIB (PF3 e PF2 por ser certificada de acordo com as normas citadas) adota processos organizacionais relacionados à capacitação tecnológica (inovações incrementais em produtos, licenciamento, codesenvolvimento com comprador, etc.) que revelam capacitação tecnológica intermediária.

P3: uma empresa que está num nível avançado de capacitação tecnológica em TIB (PF1 por ser certificada de acordo com as normas citadas) adota processos organizacionais relacionados à capacitação tecnológica (inovações radicais em produtos e processos) que revelam capacitação avançada.

Os indicadores criados para mensurar o nível de capacidade tecnológica das empresas foram extraídos a partir da matriz de Lall (1992) e suas definições encontram-se no Quadro 4.

\subsection{Método de coleta de informações}

Para a obtenção de informações, foi realizado um survey, com questionários enviados para uma amostra de 103 empresas de diferentes níveis de capacidade tecnológica do setor metal-mecânico, localizadas predominantemente no Estado de São Paulo. Essas empresas foram identificadas a partir de bancos de dados de uma instituição certificadora de sistemas de gestão da qualidade e de um instituto de pesquisas ligado a um centro universitário. Com isso, havia a garantia de que as empresas tinham certificação de algum tipo; evidentemente, havia empresas com vários tipos de certificação.

A escolha do setor metal-mecânico é justificada pelas seguintes razões:

- Entre as empresas brasileiras que inovam e diferenciam produtos, o setor em questão ocupa a liderança com $31,6 \%$ do total das empresas industriais, seguido do setor químico com $22,5 \%$ e do eletrônico com 12,9\%, segundo Kupfer e Rocha (2005);

- Juntamente com os setores de material de transporte, químico, agroindústria, eletrônico e combustíveis, concentra a maior parte da geração de comércio exterior da indústria brasileira (ibid).

Trata-se, portanto, de um setor relativamente dinâmico e que proporciona um terreno fértil para a prospecção de dados visando alcançar o objetivo final deste trabalho de pesquisa.

\section{Resultados}

Para se chegar aos resultados desta pesquisa, foram utilizadas as seguintes técnicas estatísticas: técnica da correlação canônica com significância de $5 \%$ para se concluir sobre as relações significativas entre TIB e capacidade tecnológica; e a técnica de análise de correspondência ANACOR para se concluir sobre a variação na utilização dos mecanismos de aprendizagem em TIB durante o seu processo de formação de competências.

\subsection{Resultados encontrados sobre as relações entre TIB e capacidade tecnológica}

Com a aplicação da técnica da correlação canônica, chegou-se aos valores sintetizados no Quadro 5. O 
Quadro 2. Perfis metrológico, normativo e conformativo de empresas.

\begin{tabular}{|c|c|c|c|}
\hline $\begin{array}{c}\text { Níveis em } \\
\text { TIB }\end{array}$ & Metrologia & Normalização & Avaliação da conformidade \\
\hline Primário & $\begin{array}{l}\text { Medições de baixa complexidade } \\
\text { técnica realizadas sem } \\
\text { procedimentos padronizados } \\
\text { em ambientes não controlados e } \\
\text { utilizando-se de instrumentos não } \\
\text { rastreados a padrões de medidas } \\
\text { nacionais e/ou internacionais. } \\
\text { Ausência de confiabilidade } \\
\text { metrológica. }\end{array}$ & $\begin{array}{l}\text { Ausência de infraestrutura de apoio } \\
\text { à utilização de normas técnicas na } \\
\text { empresa; não utilização voluntária } \\
\text { de normas; inexistência de acervo } \\
\text { de normas e/ou regulamentos } \\
\text { técnicos; eventual utilização } \\
\text { somente de normas técnicas de } \\
\text { características de produtos. }\end{array}$ & $\begin{array}{l}\text { Avaliação pontual da } \\
\text { conformidade, de } 1^{a} \text { parte, } \\
\text { com teste de funcionamento } \\
\text { apenas do principal requisito } \\
\text { a ser atendido pelo produto. }\end{array}$ \\
\hline $\begin{array}{l}\text { Básico } \\
\text { (PF5) }\end{array}$ & \begin{tabular}{|c|} 
Medições realizadas com \\
procedimentos padronizados, \\
em ambiente controlado, por \\
técnicos treinados e utilizando-se \\
de instrumentos/sistemas de \\
medição calibrados periodicamente \\
diante de padrões nacionais/ \\
internacionais adequados, e \\
considerados como aceitos perante \\
critérios de aceitabilidade definidos \\
previamente. Atendimento ao item \\
7.6 da norma ISO 9001.
\end{tabular} & $\begin{array}{l}\text { Capacidade para utilização } \\
\text { voluntária de normas de } 1^{\mathrm{a}} \text { geração } \\
\text { (genéricas), p.ex.: ISO } 9001 .\end{array}$ & $\begin{array}{l}\text { Certificação do Sistema } \\
\text { de Gestão da Qualidade } \\
\text { segundo a ISO 9001; } \\
\text { avaliação da conformidade } \\
\text { com tratamento } \\
\text { sistêmico - envolvendo } \\
\text { testes de performance do } \\
\text { produto de } 1^{\text {a }} \text { ou } 2^{\mathrm{a}} \text { parte. }\end{array}$ \\
\hline $\begin{array}{c}\text { Extrabásico } \\
\text { (PF4) }\end{array}$ & $\begin{array}{c}\text { Utilização da metodologia MSA } \\
\text { (Análise do Sistema de Medição) } \\
\text { para proporcionar o aumento de } \\
\text { confiança nas leituras obtidas e } \\
\text { verificação de adequação ao uso dos } \\
\text { instrumentos de medir, por meio de } \\
\text { técnicas estatísticas. }\end{array}$ & $\begin{array}{c}\text { Capacidade para utilização } \\
\text { das normas de } 2^{\text {a }} \text { geração } \\
\text { (setor específico) e de } 3^{\text {a }} \\
\text { geração (normas de empresas), } \\
\text { de grande influência em setores } \\
\text { técnicos específicos, p.ex.: TS } \\
16949 \text { (QS 9000); utilização } \\
\text { das técnicas de FMEA, APQP e } \\
\text { PPAP; utiliz. da norma ISO 17050 } \\
\text { (INTERNATIONAL..., 2004b). }\end{array}$ & $\begin{array}{l}\text { Capacidade de utilização } \\
\text { do mecanismo "Declaração } \\
\text { da Conformidade pelo } \\
\text { Fornecedor" de avaliação da } \\
\text { conformidade, de } 1^{a} \text { parte; } \\
\text { Certificação ISO TS } 16949 \\
\text { ou de outro setor específico. }\end{array}$ \\
\hline $\begin{array}{c}\text { Intermediário } \\
\text { (PF3) }\end{array}$ & $\begin{array}{l}\text { Criação de um laboratório de } \\
\text { metrologia acreditado com } \\
\text { possibilidade de prestar serviços } \\
\text { externos, pertencente à Rede } \\
\text { Brasileira de Calibração - RBC. }\end{array}$ & $\begin{array}{c}\text { Capacidade para utilização } \\
\text { voluntária da norma NBR ISO/ } \\
\text { IEC } 17025 \text { - Requisitos para a } \\
\text { competência de laboratórios de } \\
\text { ensaio e calibração. } \\
\text { Capacidade para utilização antecipada } \\
\text { de normas técnicas internacionais para } \\
\text { os seus produtos/processos tendo o } \\
\text { mercado mundial como referência. }\end{array}$ & $\begin{array}{l}\text { Acreditação de um } \\
\text { laboratório de ensaios } \\
\text { prestador de serviços } \\
\text { externos e pertencente } \\
\text { à RBLE; certificação } \\
\text { voluntária de produto por } \\
\text { um agente de } 3^{\text {a }} \text { parte. }\end{array}$ \\
\hline $\begin{array}{l}\text { Intermediário } \\
\text { Superior } \\
\text { (PF2) }\end{array}$ & $\begin{array}{c}\text { Automação de equipamentos de } \\
\text { medição, tanto na linha de produção } \\
\text { como no laboratório de metrologia. }\end{array}$ & $\begin{array}{c}\text { Capacidade para utilização } \\
\text { voluntária das normas ambientais } \\
\text { e/ou sociais, p. ex.: ambiental } \\
\text { ISO 14001, segurança/saúde } \\
\text { ocupacional (BRITISH..., 2007), } \\
\text { responsabilidade social. }\end{array}$ & $\begin{array}{c}\text { Certificação do(s) } \\
\text { Sistema(s) de Gestão } \\
\text { Ambiental segundo a ISO } \\
14001 \text { e/ou de Segurança } \\
\text { e Saúde Ocupacional } \\
\text { (BRITISH..., 2007) e/ou de } \\
\text { Responsabilidade Social. }\end{array}$ \\
\hline $\begin{array}{c}\text { Avançado } \\
\text { (PF1) }\end{array}$ & $\begin{array}{l}\text { Desenvolvimento de procedimentos } \\
\text { de medição para novas tecnologias } \\
\text { (Ex. nanousinagem, biomecânica, } \\
\text { ciência dos novos materiais, } \\
\text { nanoeletrônica, etc.) utilizando } \\
\text { equipamentos de medição de última } \\
\text { geração. }\end{array}$ & $\begin{array}{c}\text { Capacidade para influenciar } \\
\text { tecnicamente as instâncias de } \\
\text { definição de políticas para normas e } \\
\text { padrões globais } \\
\text { Participação efetiva no processo } \\
\text { de normalização regional e } \\
\text { internacional. }\end{array}$ & $\begin{array}{c}\text { Certificação de sistemas, } \\
\text { produtos e/ou serviços } \\
\text { que estejam na fronteira } \\
\text { tecnológica internacional; } \\
\text { capacidade de influenciar } \\
\text { tecnicamente e interagir } \\
\text { com o Inmetro e o comitê de } \\
\text { avaliação da conformidade } \\
\text { da ISO - ISO/Casco. }\end{array}$ \\
\hline
\end{tabular}




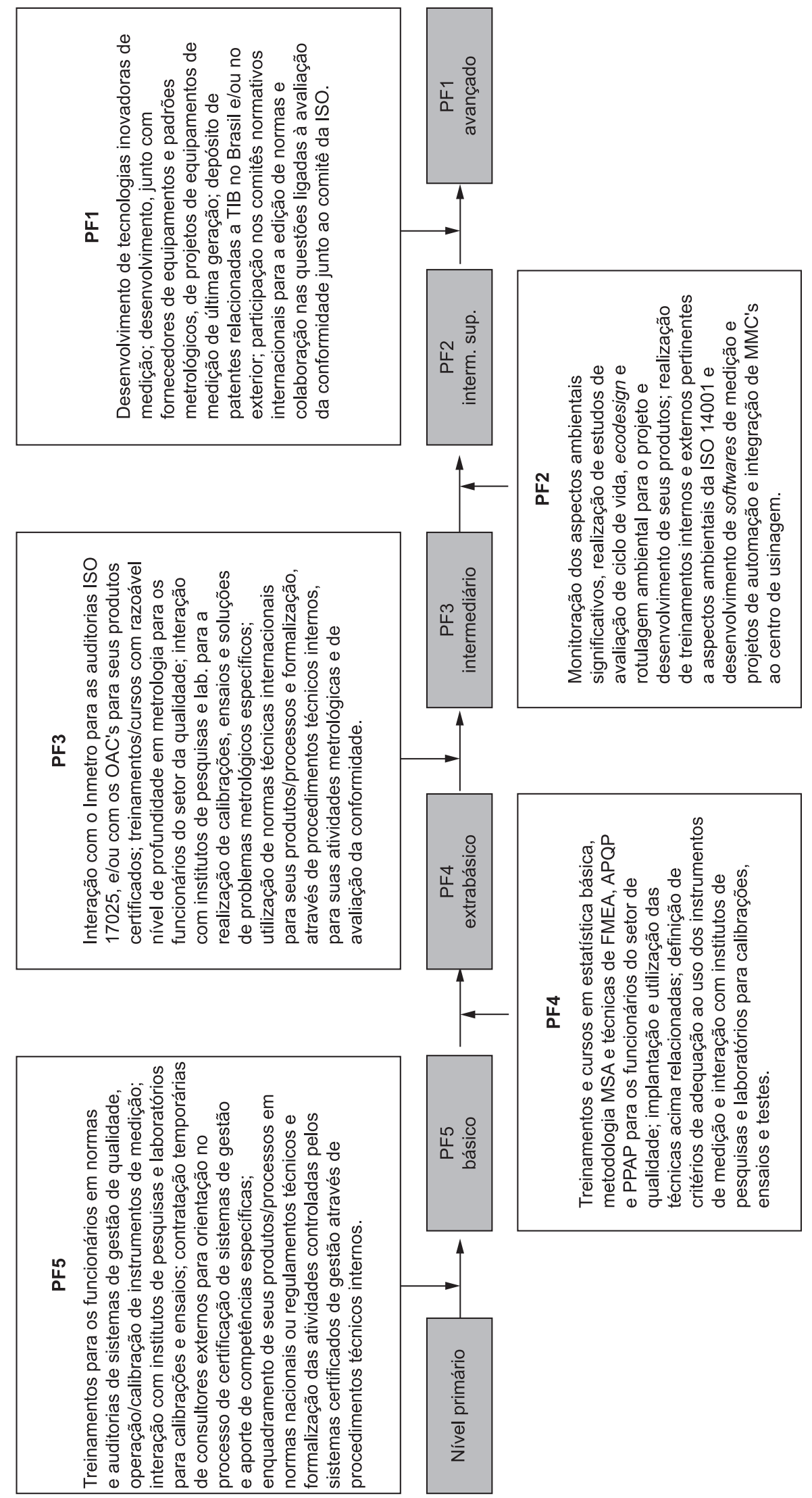

Figura 2. Principais mecanismos de aprendizagem para a formação e acumulação de competências em TIB. 
Quadro 3. Modelo conceitual da pesquisa.

\begin{tabular}{|c|c|c|}
\hline $\begin{array}{c}\text { Variável independente } \\
\text { TIB }\end{array}$ & & $\begin{array}{c}\text { Variável dependente } \\
\text { Capacidade tecnológica }\end{array}$ \\
\hline Indicadores & & Indicadores \\
\hline \multirow[t]{2}{*}{$\begin{array}{l}\text {.Certificação ISO } 9001 \text { - (PF5) } \\
\text {.Certificação TS } 16949 \text { - (PF4) }\end{array}$} & $\begin{array}{l}\text { Nível básico } \\
\text { Proposição } 1 \text { (P1) }\end{array}$ & $\begin{array}{l}\text { IND1 - Engenharia reversa } \\
\text { IND2 - Adapt. produto ao mercado } \\
\text { IND3 - Esforços em equipamentos } \\
\text { IND4 - Relação com fornecedores } \\
\text { IND5 - Controle da qualidade }\end{array}$ \\
\hline & Nível intermediário & \\
\hline \multirow[t]{2}{*}{$\begin{array}{l}\text {.Certificação ISO } 17025 \text { - (PF3) } \\
\text {.Certificação de produto - (PF3) } \\
\text {.Certificação ISO } 14001 \text { - (PF2) } \\
\text {.Automação de medições - (PF2) }\end{array}$} & Proposição 2 (P2) & $\begin{array}{l}\text { IND6 - Inovações increm. produtos } \\
\text { IND7 - Adapt. increm. nos processos } \\
\text { IND8 - Licenciam. de tecnologias } \\
\text { IND9 - Codesenv. com fornecedor } \\
\text { IND10 - Competências organiz. } \\
\text { IND11 - Cooperação em C\&T }\end{array}$ \\
\hline & Nível avançado & \\
\hline $\begin{array}{c}\text {.Participação em comitês normativos } \\
\text { internacionais - (PF1) } \\
\text {.Sistemas inovadores de } \\
\text { medição - (PF1) }\end{array}$ & Proposição 3 (P3) & $\begin{array}{l}\text { IND12 - Inov. radicais no processo } \\
\text { IND13 - Inov. radicais no produto } \\
\text { IND14 - Esforços em P\&D }\end{array}$ \\
\hline
\end{tabular}

IND5 não foi considerado, pois não se mostrou como um indicador com poder discriminatório.

Se levarmos em consideração que as proposições P1, $\mathrm{P} 2$ e P3 relacionam de forma positiva os indicadores de TIB com os indicadores de CT nos níveis básico, intermediário e avançado, respectivamente, era de se esperar que ocorressem relações estatisticamente significativas entre esses indicadores dentro dos três níveis considerados. E, de fato, a pesquisa revelou que os valores estatisticamente significativos mostraram uma tendência de distribuição ao longo da área sombreada do Quadro 5, que representa o local das relações significativas esperadas.

\subsubsection{A análise da proposição 1 (P1)}

Diante dos resultados encontrados, podemos concluir que foi encontrada associação significativa entre os indicadores ISO 9001 (INTERNATIONAL..., 2008) e TS 16949 (INTERNATIONAL..., 2009) com o IND1 (atividades de engenharia reversa).

O que podemos depreender desse resultado é que as empresas em questão, por estarem ainda num nível básico de capacidade e, consequentemente, iniciando sua escalada em busca de melhorias de qualidade em seus produtos, encontrem na engenharia reversa uma das formas de atingir esse fim. Com a conquista da certificação de seu sistema de gestão da qualidade segundo a ISO 9001 (INTERNATIONAL..., 2008), a empresa passa a dispensar um tratamento sistêmico à avaliação da conformidade de seus produtos e processos. Esse tratamento sistêmico adota o modelo PDCA (planejar, fazer, verificar e agir), estabelecendo ações que contemplam desde a seleção da norma técnica adequada, passando pela fase de engenharia do produto/engenharia reversa, até o acompanhamento do produto no mercado ao longo do tempo. Nesse sentido, podemos considerar que a ISO 9001 (INTERNATIONAL..., 2008) e também a TS 16949 (INTERNATIONAL..., 2009), sendo indutoras desse processo de melhoria contínua e tendo a empresa evoluído na questão metrológica, motivada pelas exigências dessas mesmas normas, representam um forte indicativo de contribuição para a melhoria da capacidade tecnológica da empresa.

\subsubsection{A análise da proposição 2 (P2)}

Diante dos resultados encontrados, podemos concluir que foi encontrada associação significativa entre os indicadores ISO 17025 (INTERNATIONAL..., 2005), certificação de produtos, ISO 14001 (INTERNATIONAL..., 2004a) e automação de medições com os indicadores IND6 (inovações incrementais nos produtos), IND8 (licenciamento de tecnologias) e IND10 (competências organizacionais - prática de JIT, Kaizen, TQC/ TQM e uso de sistemas integrados de informação).

Diante desse resultado, podemos inferir que uma empresa que é certificada ISO 17025 (INTERNATIONAL..., 2005) - norma que trata de competência de laboratórios de ensaio e calibração -, e que, portanto, tem um grande conhecimento metrológico, consegue projetar e garantir produtos confiáveis e certificados por rígidas normas internacionais, pois seu sistema de 
Quadro 4. Indicadores criados para capacidade tecnológica.

\begin{tabular}{|c|c|c|c|}
\hline Nível & Indicadores & Definição dos indicadores & Escala \\
\hline \multirow{5}{*}{ 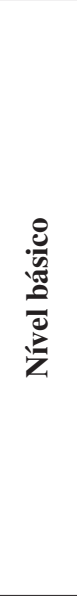 } & $\begin{array}{l}\text { Eng. Reversa } \\
\text { (IND1) }\end{array}$ & Indica se a empresa realiza ou não engenharia reversa. & $\begin{array}{c}0 \text { ou } 1 \\
\text { dummy }\end{array}$ \\
\hline & $\begin{array}{l}\text { Adaptações às necessidades } \\
\text { do mercado (AM) } \\
\text { (IND2) }\end{array}$ & $\begin{array}{l}\text { Indica se a empresa realiza pequenas adaptações nos seus produtos em } \\
\text { função das necessidades do mercado, quando solicitada. }\end{array}$ & $\begin{array}{l}0 \text { ou } 1 \\
\text { dummy }\end{array}$ \\
\hline & $\begin{array}{l}\text { Esforços em } \\
\text { equipamentos } \\
\text { (IND3) }\end{array}$ & $\begin{array}{l}\text { O indicador mensura a realização pela empresa das seguintes atividades: } \\
\text { desenvolvimento de equipamentos para a utilização na planta; fabricação } \\
\text { de peças de reposição quando necessário; treinamento de recursos } \\
\text { humanos pela empresa na ocasião da aquisição de novos equipamentos; e } \\
\text { sistema de manutenção preventiva. }\end{array}$ & $\begin{array}{l}0-100 \% \\
\text { métrica }\end{array}$ \\
\hline & $\begin{array}{l}\text { Relação com } \\
\text { fornecedores } \\
\quad \text { (IND4) }\end{array}$ & $\begin{array}{l}\text { Mede a interação da empresa com fornecedores, nas seguintes questões: se } \\
\text { a empresa possui um cadastro atualizado dos fornecedores; qualificação; e } \\
\text { controle de qualidade dos fornecedores efetuado pela empresa. }\end{array}$ & $\begin{array}{l}0-100 \% \\
\text { métrica }\end{array}$ \\
\hline & $\begin{array}{l}\text { Controle da Qualidade } \\
\text { (IND5) }\end{array}$ & $\begin{array}{l}\text { Indica se a empresa possui ou não um sistema de controle da } \\
\text { qualidade. }\end{array}$ & $\begin{array}{c}0 \text { ou } 1 \\
\text { dummy }\end{array}$ \\
\hline \multirow{6}{*}{ 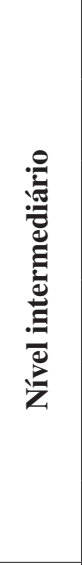 } & $\begin{array}{l}\text { Inovações incrementais } \\
\text { (IND6) }\end{array}$ & $\begin{array}{l}\text { Indica se a empresa realiza ou não inovações incrementais nos seus } \\
\text { produtos próprios ou licenciados. }\end{array}$ & $\begin{array}{c}0 \text { ou } 1 \\
\text { dummy }\end{array}$ \\
\hline & $\begin{array}{l}\text { Adapt. increm. processos } \\
\text { (IND7) }\end{array}$ & $\begin{array}{l}\text { Indica se a empresa realiza ou não adaptações incrementais em seus } \\
\text { processos. }\end{array}$ & $\begin{array}{c}0 \text { ou } 1 \\
\text { dummy }\end{array}$ \\
\hline & $\begin{array}{l}\text { Licenciamento de } \\
\text { tecnologias } \\
\text { (IND8) }\end{array}$ & $\begin{array}{l}\text { Indica se a empresa realiza ou não licenciamento de tecnologias de } \\
\text { terceiros para seus processos. }\end{array}$ & $\begin{array}{l}0 \text { ou } 1 \\
\text { dummy }\end{array}$ \\
\hline & $\begin{array}{l}\text { Codesenvolvimentos } \\
\text { (IND9) }\end{array}$ & $\begin{array}{l}\text { Indica se a empresa realiza ou não codesenvolvimentos de produtos/ } \\
\text { processos com seus fornecedores. }\end{array}$ & $\begin{array}{c}0 \text { ou } 1 \\
\text { dummy }\end{array}$ \\
\hline & $\begin{array}{l}\text { Competências } \\
\text { Organizacionais } \\
\text { (IND10) }\end{array}$ & $\begin{array}{l}\text { Esse indicador mensura se a empresa faz uso de: just in time; Kaizen; } \\
\text { TQC ou TQM; arranjos físicos adaptáveis à linha de produção; e } \\
\text { sistemas integrados de informação. }\end{array}$ & $\begin{array}{l}0-100 \% \\
\text { métrica }\end{array}$ \\
\hline & $\begin{array}{l}\text { Cooperação em C\&T } \\
\text { (IND11) }\end{array}$ & $\begin{array}{l}\text { Indica se a empresa realiza ou não convênios de cooperação científica e } \\
\text { tecnológica com universidades e/ou institutos de pesquisas. }\end{array}$ & $\begin{array}{c}0 \text { ou } 1 \\
\text { dummy }\end{array}$ \\
\hline \multirow{3}{*}{ 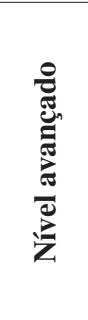 } & $\begin{array}{l}\text { Inov. radicais no processo } \\
\text { (IND12) }\end{array}$ & $\begin{array}{l}\text { Indica se a empresa realiza ou não inovações radicais no processo } \\
\text { produtivo. }\end{array}$ & $\begin{array}{c}0 \text { ou } 1 \\
\text { dummy }\end{array}$ \\
\hline & $\begin{array}{l}\text { Inov. radicais no produto } \\
\text { (IND13) }\end{array}$ & $\begin{array}{l}\text { Indica se a empresa realiza ou não inovações radicais na engenharia do } \\
\text { produto. }\end{array}$ & $\begin{array}{c}0 \text { ou } 1 \\
\text { dummy }\end{array}$ \\
\hline & $\begin{array}{l}\text { Esforços em P\&D } \\
\text { (IND14) }\end{array}$ & $\begin{array}{l}\text { Esse indicador mensura os esforços realizados pela empresa nas } \\
\text { seguintes questões: requerimento de patentes no Brasil e no exterior; } \\
\text { se possui depto. próprio de P\&D; e se licencia suas próprias } \\
\text { tecnologias para terceiros. }\end{array}$ & $\begin{array}{l}0-100 \% \\
\text { métrica }\end{array}$ \\
\hline
\end{tabular}

medição é reconhecido internacionalmente por sua competência. Uma empresa com esse perfil consegue introduzir inovações incrementais em seus produtos com maior facilidade. Acrescenta-se a isso o fato de que a automação de um processo de medição garante uma taxa maior de confiabilidade das medições e, consequentemente, na melhoria da qualidade do produto final.

Já a integração de uma máquina de medição de coordenadas (MMC) com o centro de usinagem, além de permitir a automação de resultados, melhora a performance no equipamento de medição. Uma MMC permite uma informação rápida e precisa com um sistema de retroalimentação que transmite os dados obtidos na medição por coordenadas para o centro de usinagem, corrigindo a programação e melhorando a exatidão do produto. Nesse caso, torna-se evidente a contribuição da metrologia num processo de inovação incremental nos produtos e no uso de sistemas integrados de informação.

No caso da certificação ambiental ISO 14001 (INTERNATIONAL..., 2004a), esta reflete um alto grau de amadurecimento com relação à consciência ecológica e ao potencial tecnológico da empresa. Essas empresas são líderes de cadeias de fornecimento ou simplesmente participam delas. Como condição básica para participarem dessas cadeias há a necessidade de se estruturarem para realizar eco-design, avaliação de ciclo de vida em seus produtos, rotulagem ambiental, práticas de just-in-time, Kaizen, TQC/TQM, sistemas integrados de informação e facilidade de adaptar seus 
arranjos físicos à linha de produção, entre outras atividades.

Podemos considerar, a partir daí, que a ISO 17025 (INTERNATIONAL..., 2005), a certificação de produtos, a ISO 14001 (INTERNATIONAL..., 2004a) e a automação dos processos de medição representam um indicativo de que são importantes na efetiva contribuição com o incremento da capacidade tecnológica das empresas.

\subsubsection{A análise da proposição 3 (P3)}

Foi encontrada associação significativa entre os indicadores - participação em comitês normativos internacionais e utilização de sistemas inovadores de medição - com o IND14 (esforços em P\&D).
Uma empresa que participa ativamente de comitês normativos internacionais, influenciando tecnologicamente suas decisões e/ou que possui sistemas inovadores de medição, consegue sucesso em seus esforços em $\mathrm{P} \& \mathrm{D}$, como depositar patentes, possuir departamentos próprios de $\mathrm{P} \& \mathrm{D}$ e licenciar suas próprias tecnologias para terceiros.

Podemos considerar, a partir daí, que a participação em comitês normativos internacionais e a existência de sistemas inovadores de medição representam um indicativo de que são pontos importantes para a efetiva contribuição com o incremento da capacidade tecnológica das empresas.

Diante das análises dessas três proposições e da tendência revelada pelo Quadro 5 de distribuição dos valores estatisticamente significativos ao longo da

Quadro 5. Resumo dos valores de significâncias encontradas.

\begin{tabular}{|c|c|c|c|c|c|c|c|}
\hline & \multicolumn{5}{|c|}{ Indicadores de TIB } \\
\hline & & & ISO 9001 & TS 16949 & $\begin{array}{c}\text { ISO 17025; } \\
\text { Certificação de } \\
\text { produtos }\end{array}$ & $\begin{array}{c}\text { ISO 14001; } \\
\text { Automação } \\
\text { das medições }\end{array}$ & $\begin{array}{c}\text { Particip. em comitês } \\
\text { normativos internac.; } \\
\text { Sistemas inovadores de } \\
\text { medição }\end{array}$ \\
\hline \multirow{3}{*}{\multicolumn{2}{|c|}{ : }} & IND14 & 0,513 & 0,340 & 0,161 & 0,174 & $0,022 *$ \\
\hline & & IND13 & 0,250 & 0,912 & 0,887 & $0,034 *$ & 0,145 \\
\hline & & IND12 & 0,198 & 0,532 & 0,084 & $0,012 *$ & 0,906 \\
\hline$\stackrel{0}{0}$ & \multirow{6}{*}{ 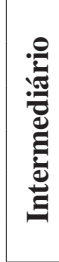 } & IND11 & 0,642 & 0,357 & 0,239 & 0,627 & $0,014 *$ \\
\hline$\stackrel{\Xi}{0}$ & & IND10 & 0,677 & 0,094 & 0,071 & $0,001 *$ & 0,411 \\
\hline ت్ & & IND9 & 0,934 & 0,526 & 0,360 & 0,946 & 0,887 \\
\hline 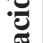 & & IND8 & 0,340 & 0,251 & $0,041 *$ & 0,849 & 0,588 \\
\hline ق & & IND7 & 0,756 & 0,151 & 0,825 & 0,833 & 0,559 \\
\hline 용 & & IND6 & 0,885 & 0,577 & $0,024 *$ & 0,773 & 0,606 \\
\hline \multirow{4}{*}{ 管 } & \multirow{4}{*}{ 选 } & IND4 & 0,208 & 0,978 & 0,288 & 0,878 & 0,203 \\
\hline & & IND3 & 0,808 & 0,997 & 0,332 & $0,029 *$ & 0,846 \\
\hline & & IND2 & 0,528 & 0,183 & 0,646 & 0,337 & 0,946 \\
\hline & & IND1 & $0,050 *$ & $0,043 *$ & 0,500 & $0,010^{*}$ & 0,792 \\
\hline
\end{tabular}

(*) Relações que se mostraram significativas no nível de $5 \%$.

Quadro 6. Síntese das relações estatisticamente significativas encontradas.

\begin{tabular}{|l|l|}
\hline \multicolumn{1}{|c|}{ Indicador TIB } & \multicolumn{1}{|c}{ Indicador de Capacidade Tecnológica } \\
\hline ISO 9001 (PF5) & \multirow{2}{*}{ Prática de engenharia reversa (IND1) } \\
\hline TS 16949 (PF4) & \\
\cline { 2 - 2 } & Esforços em equipamentos (IND3) \\
\cline { 2 - 2 } ISO 14001 e/ou automações das medições (PF2) & Competências Organizacionais (IND10) \\
\cline { 2 - 2 } & Inovações radicais no processo (IND13) \\
\cline { 2 - 2 } & Inovações radicais no produto (IND12) \\
\hline \multirow{2}{*}{ ISO 17025 e/ou certif. de produtos (PF3) } & Inovações incrementais no produto (IND6) \\
\cline { 2 - 2 } $\begin{array}{l}\text { Sistemas inovadores de medição e/ou participação em } \\
\text { comitês normativos internacionais (PF1) }\end{array}$ & Licenciamento de tecnologias de terceiros (IND8) \\
\cline { 2 - 2 } & Cooperação em C\&T (IND11) \\
\hline
\end{tabular}


área sombreada que representa o local das relações significativas esperadas, pode-se concluir que há fortes indícios de que a TIB exerce contribuição na formação e acumulação das competências tecnológicas das empresas nos três níveis considerados - básico, intermediário e avançado.

E finalmente, um resumo das relações significativas encontradas entre os indicadores de TIB e os indicadores de capacidade tecnológica pode ser observado no Quadro 6.

\subsection{Resultados encontrados sobre os mecanismos de aprendizagem}

Com o intuito de se responder à segunda questão de pesquisa deste trabalho, foi solicitado às empresas respondentes que selecionassem, no questionário de pesquisa, entre os cinco perfis ali descritos (PF5, PF4, PF3, PF2 e PF1), aquele que mais se aproximava dos mecanismos de aprendizagem em TIB praticados pela empresa.

Como era conhecido o nível em TIB que as empresas efetivamente possuem, pelas suas certificações declaradas no questionário (considerado como valor verdadeiro de resposta), foi testada a concordância com aquilo que foi respondido pelas empresas em relação ao perfil atribuido (valor observado de resposta). Havendo concordância, depreende-se que há variação dos mecanismos de aprendizagem, pois as certificações vão sendo conquistadas ao longo do tempo. Ou seja, quanto mais próximos os valores real (nível em TIB que a empresa efetivamente tem) e observado, mais significativa é a condição de variação dos mecanismos de aprendizagem em TIB.

A aplicação da técnica ANACOR, precedida de um teste quiquadrado que visa verificar a dependência das variáveis, revelou que os valores encontrados como respostas das 103 empresas participantes da pesquisa mostraram-se associados entre si, ou seja, são dependentes e não se distribuem de forma aleatória, conforme pode-se notar no Quadro 7 e no mapa perceptual da Figura 3.

Tendo em vista o resultado encontrado do teste quiquadrado para o nível de significância praticamente nulo, conclui-se que há associação entre as frequências observada (respondido pela empresa) e esperada (real). Portanto, esse resultado indica a existência da dependência entre as duas variáveis e, consequentemente, a adequação da aplicação da ANACOR.

Na sequência, aplicando-se a técnica da ANACOR, chegou-se ao mapa perceptual da Figura 3.

Constata-se, por esse mapa, que, pelo fato de as categorias das variáveis (PF observado e PF real) estarem dispostas próximas umas das outras, existe associação entre PF1 obs/PF1 real, PF2 obs/PF2 real, PF3 obs/PF3 real, PF4 obs/PF4 real e PF5 obs/PF5 real.

Portanto, diante desses resultados, podemos concluir que os mecanismos de aprendizagem em TIB utilizados pelas empresas variam ao longo dos diversos estágios de formação e acumulação de suas capacidades, de acordo com o referencial analítico sintetizado na Figura 2.

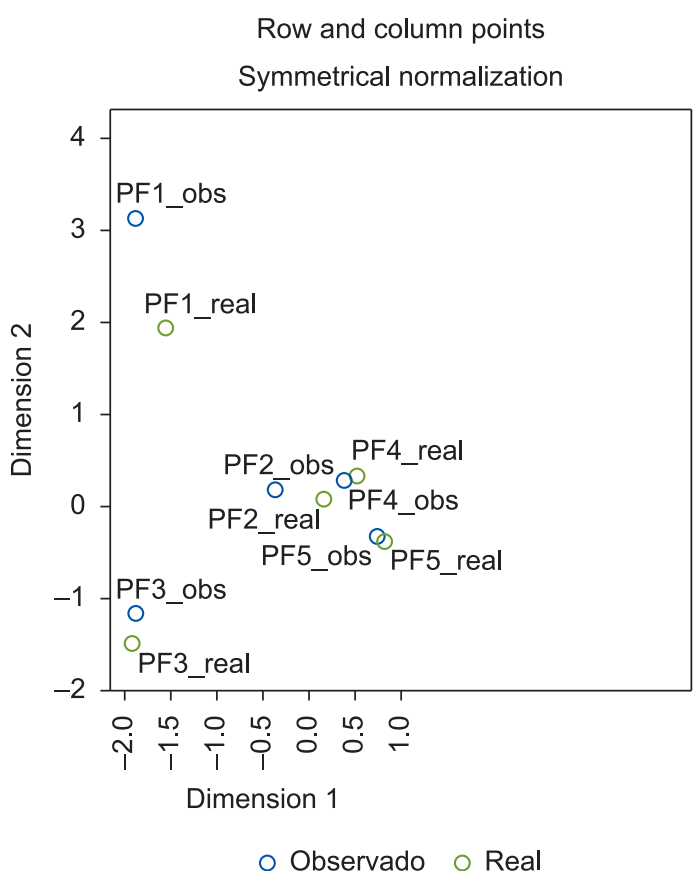

Figura 3. Mapa perceptual.

Quadro 7. Resultado do teste quiquadrado.

\begin{tabular}{|c|c|c|c|c|c|c|c|c|}
\hline \multicolumn{4}{|c|}{} & \multicolumn{2}{c|}{$\begin{array}{c}\text { Proporção da } \\
\text { inércia }\end{array}$} & \multicolumn{2}{c|}{$\begin{array}{c}\text { Confiança do valor } \\
\text { singular }\end{array}$} \\
\hline Dimensão & Valor singular & Inércia & $\begin{array}{c}\text { Qui- } \\
\text { quadrado }\end{array}$ & Significância & Valor & $\begin{array}{c}\text { Valor } \\
\text { Acumulado }\end{array}$ & $\begin{array}{c}\text { Desvio } \\
\text { Padrão }\end{array}$ & $\begin{array}{c}\text { Correlação } \\
\mathbf{2}\end{array}$ \\
\hline 1 & 0,828 & 0,686 & & & 0,453 & 0,453 & 0,047 & 0,313 \\
\hline 2 & 0,619 & 0,384 & & & 0,253 & 0,706 & 0,112 & \\
\hline 3 & 0,581 & 0,338 & & & 0,223 & 0,929 & & \\
\hline 4 & 0,328 & 0,108 & & & 0,071 & 1,000 & & \\
\hline Total & & 1,516 & 156,130 & $0,000^{\mathrm{a}}$ & 1,000 & 1,000 & & \\
\hline
\end{tabular}

${ }^{\mathrm{a}} 16$ graus de liberdade. 
Quadro 8. Matriz de Lall (1992) adaptada à Tecnologia Industrial Básica.

\begin{tabular}{|c|c|c|c|c|c|c|c|}
\hline & \multicolumn{4}{|c|}{ Dimensões funcionais } & \multirow{3}{*}{$\begin{array}{l}\text { Tecnologia industrial } \\
\text { básica }\end{array}$} \\
\hline & & & \multicolumn{3}{|c|}{ Produção } & \multirow{2}{*}{$\begin{array}{l}\text { Ligações com } \\
\text { outros agentes }\end{array}$} & \\
\hline & & & Eng. de Produto & $\begin{array}{l}\text { Eng. de } \\
\text { Processo }\end{array}$ & $\begin{array}{c}\text { Eng. } \\
\text { Industrial }\end{array}$ & & \\
\hline \multirow{6}{*}{ 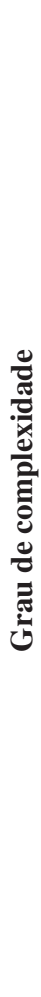 } & \multirow{3}{*}{ 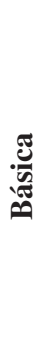 } & \multirow{3}{*}{$\begin{array}{l}\text { (baseada em } \\
\text { experiência) }\end{array}$} & Engenhariareversa & $\begin{array}{l}\text { Controle da } \\
\text { qualidade }\end{array}$ & $\begin{array}{l}\text { Estudo dos } \\
\text { métodos e } \\
\text { dos tempos } \\
\text { de trabalho }\end{array}$ & $\begin{array}{c}\text { Obtenção } \\
\text { local de bens e } \\
\text { serviços }\end{array}$ & \multirow[t]{3}{*}{ Certificação ISO 9001} \\
\hline & & & \multirow{2}{*}{$\begin{array}{c}\text { Pequenas } \\
\text { adaptações às } \\
\text { necessida-des do } \\
\text { mercado. }\end{array}$} & $\begin{array}{c}\text { Manuten-ção } \\
\text { preventiva }\end{array}$ & \multirow{2}{*}{$\begin{array}{l}\text { Controle de } \\
\text { estoque. }\end{array}$} & \multirow{2}{*}{$\begin{array}{c}\text { Troca de } \\
\text { informa-ções } \\
\text { com fornecedo- } \\
\text { res. }\end{array}$} & \\
\hline & & & & $\begin{array}{c}\text { Assimila-ção } \\
\text { de tecnologia } \\
\text { de processo. }\end{array}$ & & & \\
\hline & \multirow{2}{*}{ } & \multirow{2}{*}{$\begin{array}{l}\text { Adaptável } \\
\text { Duplicável }\end{array}$} & $\begin{array}{l}\text { Melhoria da } \\
\text { qualidade dos } \\
\text { produtos }\end{array}$ & $\begin{array}{l}\text { Licencia- } \\
\text { mento } \\
\text { de novas } \\
\text { tecnologias }\end{array}$ & $\begin{array}{l}\text { Monitora- } \\
\text { mento da } \\
\text { produtivi- } \\
\text { dade }\end{array}$ & $\begin{array}{l}\text { Transfe-rência } \\
\text { de tecnologia } \\
\text { de fornecedo- } \\
\text { res locais }\end{array}$ & $\begin{array}{l}\text { Certificação de produtos } \\
\text { por agentes de } 3^{\text {a }} \text { parte }\end{array}$ \\
\hline & & & $\begin{array}{l}\text { Modifica-ção } \\
\text { de produtos } \\
\text { adquiridos por } \\
\text { licencia-mento. }\end{array}$ & $\begin{array}{l}\text { Adaptação } \\
\text { de processos } \\
\text { e redução de } \\
\text { custos. }\end{array}$ & $\begin{array}{c}\text { Melhorias } \\
\text { na coordena- } \\
\text { ção dos } \\
\text { processos. }\end{array}$ & $\begin{array}{l}\text { Relações com } \\
\text { instituições de } \\
\text { C\&T. }\end{array}$ & $\begin{array}{c}\text { Certificação ISO } 14001 \\
\text { e/ou social } \\
\text { Automação dos } \\
\text { equipamentos de } \\
\text { medição. }\end{array}$ \\
\hline & 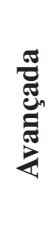 & $\begin{array}{l}\text { Inovador } \\
\text { (baseada na } \\
\text { pesquisa) }\end{array}$ & $\begin{array}{l}\text { Inovação de } \\
\text { produto in-house }\end{array}$ & $\begin{array}{c}\text { Inovação } \\
\text { de processo } \\
\text { in-house }\end{array}$ & & $\begin{array}{l}\text { Licencia-mento } \\
\text { de tecnologias } \\
\text { próprias para } \\
\text { outros }\end{array}$ & $\begin{array}{l}\text { Sistemas inovadores de } \\
\text { medição } \\
\text { Participação em } \\
\text { comitês normativos } \\
\text { internacionais. }\end{array}$ \\
\hline
\end{tabular}

\section{Conclusões}

As conclusões deste trabalho de pesquisa, quando analisadas nos três níveis de CT considerados, fornecem uma contribuição teórica para o entendimento da colaboração da TIB na formação e acumulação das capacidades tecnológicas das empresas.

Em sendo assim, tendo a matriz das capacidades tecnológicas de Lall (1992) como principal referência deste trabalho de pesquisa, agregamos nossas conclusões a ela, conforme Quadro 8.

No nível básico de capacidade tecnológica (CT), caracterizado pelas atividades de simples rotina baseada na experiência adquirida pela empresa em esforços do tipo by doing, as certificações de $1^{\mathrm{a}}$ e $2^{\mathrm{a}}$ gerações, representadas respectivamente pela ISO 9001 (INTERNATIONAL..., 2008) e TS 16949 (INTERNATIONAL..., 2009), revelaram-se vetores de contribuição para o incremento da CT das empresas, respondendo à primeira questão de pesquisa.

Essas constatações sobre a colaboração da TIB na formação e acumulação das CTs das empresas não foram encontradas nas teorias de Lall (1992), Figueiredo (2003) e Fleury (1991). Em sua matriz das capacidades tecnológicas, Lall (1992) coloca a engenharia reversa como uma atividade de nível básico, mas não faz nenhuma relação com as certificações ISO 9001 (INTERNATIONAL..., 2008) e TS 16949 (INTERNATIONAL..., 2009). O mesmo ocorre com Figueiredo (2003) e Fleury (1991). Todos esses autores, embora reconheçam em suas pesquisas a existência de sistemas de controle da qualidade, não examinaram as certificações dos sistemas da qualidade e todos os aspectos relacionados à TIB decorrentes das certificações, como elementos que contribuem para com a elevação do patamar tecnológico de uma empresa.

No nível intermediário de capacidade tecnológica (CT), caracterizado pelas atividades adaptáveis e duplicáveis, baseadas na busca e cujas capacidades são construídas a partir das atividades ou esforços conduzidos em base mais deliberada, a certificação ISO 17025 (INTERNATIONAL..., 2005), a certificação ISO 14001 (INTERNATIONAL..., 2004a), a certificação de produtos e a automação de processos metrológicos revelaram-se importantes elementos de contribuição para o incremento das CTs das empresas, respondendo à primeira questão de pesquisa.

Tanto Lall (1992) como Figueiredo (2003) e Fleury (1991) não citaram em seus estudos os benefícios 
decorrentes dessas certificações e da automação dos processos metrológicos para o incremento da capacidade tecnológica.

Lall (1992) coloca as atividades de inovações incrementais nos produtos; o licenciamento de tecnologias e as competências organizacionais, como atividades de nível intermediário; mas não faz nenhuma referência em relação às certificações comentadas anteriormente e as suas possíveis relações com as atividades por ele colocadas.

No nível avançado de capacidade tecnológica (CT), caracterizado por meio de atividades de P\&D - que são a forma mais explícita e deliberada de esforço - os sistemas inovadores de medição e a influência tecnológica junto aos comitês normativos da ISO revelaram-se importantes componentes de contribuição para o incremento da CT das empresas, respondendo à primeira questão de pesquisa.

Muito embora Lall (1992) e Figueiredo (2003) tenham colocado as atividades de P\&D no estágio avançado de capacidade tecnológica que uma empresa pode ocupar, nenhum desses autores fez referências ao apoio metrológico às inovações radicais - tanto em processos como em produtos.

Pelo que pudemos constatar, de uma forma geral, os principais autores e pesquisadores de aprendizagem tecnológica, entre os quais Dosi (1998), Fleury (1991), Lall (1992), Bell e Pavitt (1995), Kim (1997) e Figueiredo (2003), não consideraram o papel da TIB na formação e acumulação das capacidades tecnológicas.

Não foram encontrados, em suas teorias, aspectos metrológicos, normativos ou até mesmo conformativos que pudessem influir nos processos de aprendizagem tecnológica das empresas.

Já com relação aos mecanismos de aprendizagem em TIB, pudemos notar, no mapa da Figura 3, que variam ao longo dos diversos estágios de formação e acumulação de suas capacidades, conforme sintetizado na Figura 2, respondendo à segunda questão de pesquisa.

$\mathrm{E}$, finalmente, devemos considerar esses resultados com algumas ressalvas, pois a pesquisa foi conduzida apenas no setor metal-mecânico e a limitação principal foi o tamanho da amostra.

\section{Referências}

BELL, M. "Learning" and the accumulation of industrial technological capacity in developing countries. In: KING, K.; FRANSMAN, M. (Eds.). Technological capability in the Third World. London: Macmilan, 1984.

BELL, M.; PAVITT, K. Technological accumulation and industrial growth: contrast between developed and developing countries. Industrial and Corporate Change, v. 2, n. 2, 1993.

BELL, M.; PAVITT, K. The development of technological capabilities. In: HAQUE, I. U. Trade, technology and international competitiveness. Washington: The World Bank, 1995.

BRITISH STANDARDS INSTITUTION - BSI. OHSAS 18001: Occupational Health and Safety Management Systems - Requirements. London: British Standards Institution, 2007.
DOSI, G. The nature of the innovative process. In: DOSI, G. et al. (Eds.) Technical change and economic theory. London: Pinter, 1988.

FIGUEIREDO, P. N. Aprendizagem tecnológica e performance competitiva. Rio de Janeiro: FGV, 2003.

FIGUEIREDO, P. N. Aprendizagem tecnológica e inovação industrial em economias emergentes: uma breve contribuição para o desenho e implementação de estudos empíricos e estratégias no Brasil. Revista Brasileira de Inovação, v. 3, n. 2, jul./dez. 2004.

FLEURY, A. C. C. Política Industrial e Capacitação Tecnológica. Departamento de Engenharia de Produção, Escola Politécnica da USP, abr. 1991.

FLEURY, A. C. C. A Tecnologia Industrial Básica (TIB) como condicionante do desenvolvimento industrial na América Latina - Estudo preparado para o CEPAL. São Paulo, 2003.

FRANSMAN, M. Technological capability in the Third World: an overview and introduction to some of the issues raised in this book. In: FRANSMAN, M.; KING, K. (Eds.).Technological capability in the Third World. London: Macmillan, 1984.

INTERNATIONAL ORGANIZATION FOR STANDARDIZATION - ISO. ISO 14001: Environmental management systems - Requirements with guidance for use. Geneva: ISO, 2004a.

INTERNATIONAL ORGANIZATION FOR STANDARDIZATION - ISO. ISO/IEC 17050: Conformity Assessment - Supplier's declaration of conformity (Part 1 and Part 2). Geneva: ISO, 2004b.

INTERNATIONAL ORGANIZATION FOR STANDARDIZATION - ISO. ISO/IEC 17025: General requirements for the competence of testing and calibration laboratories. Geneva: ISO, 2005.

INTERNATIONAL ORGANIZATION FOR STANDARDIZATION - ISO. ISO 9001: Quality management systems - Requirements. Geneva: ISO, 2008.

INTERNATIONAL ORGANIZATION FOR STANDARDIZATION - ISO. ISO/TS 16949: Quality management systems - Particular requirements for the application of ISO 9001:2008 for automotive production and relevant service part organizations. Geneva: ISO, 2009.

KIM, L. Imitation to innovation: the dynamics of Korea's technological learning. Boston: Harvard Business School Press, 1997.

KUPFER, D.; ROCHA, F. Determinantes setoriais do desempenho das empresas industriais brasileiras. In: DE NEGRI, J. A.; SALERNO, M. S. (Orgs.). Inovações, padrões tecnológicos e desempenho das firmas industriais brasileiras. Brasília: IPEA, 2005.

LALL, S. Technological capabilities and industrialization. World Development, v. 20, n. 2, 1992. http://dx.doi. org/10.1016/0305-750X(92)90097-F

NADVI, K.; WALTRING, F. Making Sense of Global Standards. Gerhard Mercator Universitat Duisburg, 2002. INEF-Report, Heft 58.

SOUZA, R. D. F. Tecnologia Industrial Básica como fator de competitividade. Revista Parcerias Estratégicas, n. 8, maio 2000 .

TACLA, C. L.; FIGUEIREDO, P. N. Processos de Aprendizagem e Acumulação de Capacidades Tecnológicas: Evidências de uma Empresa de Bens de Capital no Brasil. Revista de Administração Contemporânea, v. 7,n. 3, 2003. 